\title{
Constraining the diffuse supernova axion-like-particle background with high-latitude Fermi-LAT data
}

\author{
Christopher Eckner, ${ }^{a, *}$ Francesca Calore, ${ }^{a}$ Pierluca Carenza, ${ }^{b, c}$ Maurizio Giannotti, ${ }^{d}$ \\ Joerg Jaeckel, ${ }^{e}$ Francesco Sivo ${ }^{b, c}$ and Alessandro Mirizzi ${ }^{b, c}$ \\ ${ }^{a}$ Univ. Grenoble Alpes, USMB, CNRS, LAPTh, F-74000 Annecy, France \\ ${ }^{b}$ Dipartimento Interateneo di Fisica “Michelangelo Merlin”, Via Amendola 173, 70126 Bari, Italy. \\ ${ }^{c}$ Istituto Nazionale di Fisica Nucleare - Sezione di Bari, Via Orabona 4, 70126 Bari, Italy. \\ ${ }^{d}$ Physical Sciences, Barry University, 11300 NE 2nd Ave., Miami Shores, FL 33161, USA \\ ${ }^{e}$ Institut für theoretische Physik, Universität Heidelberg, Philosophenweg 16, 69120 Heidelberg, Germany \\ E-mail: calore@lapth.cnrs.fr, pierluca.carenza@ba.infn.it, \\ eckner@lapth.cnrs.fr, MGiannotti@barry.edu, \\ jjaeckel@thphys.uni-heidelberg.de, f.sivo1@studenti.uniba.it, \\ alessandro.mirizzi@ba.infn.it
}

Core-collapse supernovae ( $\mathrm{SNe}$ ) have been identified as a promising target to probe the existence of axion-like-particles (ALPs). The cumulative signal from all past SNe events would contain an ALP component and create a diffuse flux peaked at energies $O(50) \mathrm{MeV}$. Due to their coupling to photons and the related Primakoff process, the diffuse SNe ALP flux is converted into a diffuse gamma-ray flux while traversing the magnetic field of the Milky Way. The spatial morphology of this signal is expected to follow the shape of the Galactic magnetic field lines. We perform a template-based analysis to constrain the ALP parameter space via the spatial structure of this ALP-induced diffuse gamma-ray flux using Fermi-LAT data from 12 years and an energy range from $50 \mathrm{MeV}$ to $500 \mathrm{GeV}$. We find an improvement of the upper limit on the ALP-photon coupling constant $g_{a \gamma}$ about a factor of two compared to a previous analysis solely based on the spectral shape of the signal. Our results are robust against variations in the modelling of high-latitude Galactic diffuse emission and systematic uncertainties of the LAT, and only mildly depend on the SN spectral modelling.

$37^{\text {th }}$ International Cosmic Ray Conference (ICRC 2021)

July 12 th - 23rd, 2021

Online - Berlin, Germany

\footnotetext{
${ }^{*}$ Presenter
} 


\section{Introduction}

The idea that the universe is filled with dark matter (DM) besides the known baryonic matter has become an observationally well-established fact. As concerns the nature of this substance, little is known and a great deal of well-motivated hypotheses have been published to explain its composition. Among the proposed models, the axion takes a prominent place as it solves not only the DM puzzle but it also settles the "strong CP problem" of QCD [1]. Generalisations of the axion, axion-like particles (ALPs) often occur in extensions of the Standard Model of particle physics [2] and they offer a larger flexibility regarding model building. Both axions and ALPs may explain all of the DM density of the universe [3].

In a minimal scenario, ALPs may couple to Standard Model photons via the interaction Lagrangian [4]

$$
\mathcal{L}_{a \gamma}=-\frac{1}{4} g_{a \gamma} F_{\mu \nu} \tilde{F}^{\mu v} a=g_{a \gamma} \boldsymbol{E} \cdot \boldsymbol{B} a,
$$

where $a$ denotes the ALP field strength, $\boldsymbol{E}$ the electric field of the photon and $\boldsymbol{B}$ the field strength of an external magnetic field. This kind of coupling opens the door to explore the existence of ALPs with experiments on Earth and in space. Besides the decay of an ALP into two photons, this Lagrangian allows for the conversion of ALPs into photons within an external magnetic/electrostatic field, the so-called Primakoff process [5].

The right conditions for efficient ALP-photon conversions are met during supernova explosions of massive stars. In particular, core-collapse supernovae (CCSNe) have been identified as a powerful source of axions and ALPs $[6,7]$ where the electrostatic fields of ions, protons and electrons trigger the conversion of thermal photons into ALPs leading to an ALP flux with energies $\sim O(100) \mathrm{MeV}$. The particle emission from CCSNe has already been used to constrain the viable parameter space of axions and ALPs. As a paramount example, the lack of a gamma-ray burst following the neutrino signal from the Galactic supernova SN 1987A currently places the most stringent bounds on the coupling of ALPs to photons of $g_{a \gamma}<5.3 \cdot 10^{-12} \mathrm{GeV}^{-1}$ for ALP masses $m_{a}<4 \cdot 10^{-10} \mathrm{eV}$ [8].

As we currently cannot get hold of another CCSN in the vicinity of the Earth, we target the cumulative emission from all past CCSNe events in the universe. This isotropic signal is expected to be converted back to photons within the Milky Way's magnetic field. Due to the characteristic energy of the ALP spectrum, the resulting photon flux, the diffuse SN ALP background (DSNALPB), falls in the gamma-ray spectrum of light. It can be detected as a sub-component of the diffuse gamma-ray background at high latitudes measured by the Fermi satellite. In this work, we present our efforts to improve on existing upper limits on the DSNALPB (derived in [9] exploiting only the spectral shape of the diffuse gamma-ray flux) by performing a template-based analysis to constrain the ALP parameter space via the spatial structure of this ALP-induced diffuse gamma-ray flux using Fermi-LAT data from 12 years and an energy range from $50 \mathrm{MeV}$ to $500 \mathrm{GeV}$.

\section{The spectral and spatial morphology of the DSNALPB}

We utilise the numerical simulation results of six SN explosions whose stellar progenitors have masses of $M \in\{8.8,11.2,18,25,40,70\} M_{\odot}$. The SNe events of the four lightest stars are successful CCSNe while the heaviest two mass points represent failed $\mathrm{SNe}$, i.e. those $\mathrm{SNe}$ who 

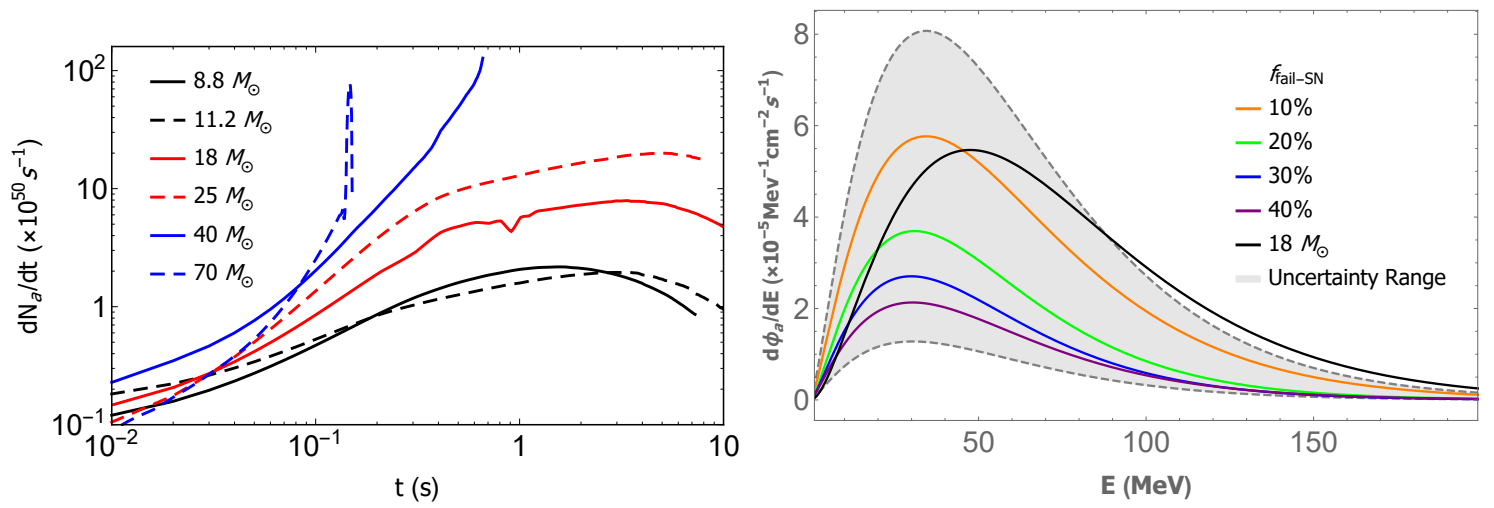

Figure 1: (Left:) Time evolution of the ALP production rate $\frac{\mathrm{d} \dot{n}_{a}}{\mathrm{~d} t}$ depending on the SN's progenitor mass. We assume $g_{a \gamma}=10^{-11} \mathrm{GeV}^{-1}$ and $m_{a} \ll 10^{-11} \mathrm{eV}$. (Right:) DSNALPB fluxes for $g_{a \gamma}=10^{-11} \mathrm{GeV}^{-1}$

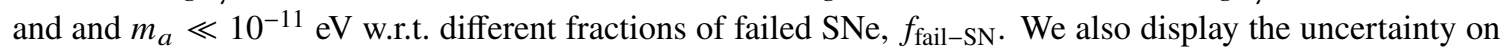
the flux due to the uncertainties on the spectral parameters and on the star-formation rate (gray band). For comparison with [9], we also show the flux obtained by assuming a monochromatic progenitor mass function at $18 M_{\odot}$.

abruptly terminate with the direct formation of a black hole. In Fig. 1, we show the time evolution of the ALP production rate $\mathrm{d} \dot{n}_{a} / \mathrm{d} t$ integrated over the SN photon energy distribution, which clearly shows the distinction between successful and failed CCSNe. Under the assumption that the ALP mass $m_{a}$ is much smaller than the temperature in the SN medium, the time-integrated ALP spectrum $\mathrm{d} N_{a} / \mathrm{d} E$ is well-approximated by the following functional form [8]

$$
\frac{d N_{a}}{d E}=C\left(\frac{g_{a \gamma}}{10^{-11} \mathrm{GeV}^{-1}}\right)^{2}\left(\frac{E}{E_{0}}\right)^{\beta} \exp \left(-\frac{(\beta+1) E}{E_{0}}\right)
$$

to which we perform fits for each SN progenitor mass. The resulting best-fit parameters are given in Tab. 1 . Hence, the cutoff energy $E_{0}$ as well as the position of the peak of the spectrum is increasingly monotonically with increasing progenitor mass.

To go from the ALP spectrum of a single CCSN event to the cumulative flux from all CCSNe throughout the history of the universe, we apply the following prescription:

$$
\frac{\mathrm{d} \phi_{a}\left(E_{a}\right)}{\mathrm{d} E_{a}}=\int_{0}^{\infty}(1+z) \frac{\mathrm{d} N_{a}^{C C}\left(E_{a}(1+z)\right)}{\mathrm{d} E_{a}} R_{S N}(z)\left|c \frac{\mathrm{d} t}{\mathrm{~d} z}\right| \mathrm{d} z
$$

where $z$ is the redshift, $R_{S N}(z)$ is the SN explosion rate taken from [10], and $|d t / d z|^{-1}=H_{0}(1+$ $z)\left[\Omega_{\Lambda}+\Omega_{M}(1+z)^{3}\right]^{1 / 2}$ with the cosmological parameters $H_{0}=67.4 \mathrm{~km} \mathrm{~s}^{-1} \mathrm{Mpc}^{-1}, \Omega_{M}=0.315$, $\Omega_{\Lambda}=0.685$ [11]. The spectrum $\mathrm{d} N_{a}^{C C} / \mathrm{d} E_{a}$ represents the ALP emission from all past SN events. We derive the latter quantity by weighing the flux from a given $\mathrm{SN}$ over the initial mass function (IMF), i.e. the number of stars formed per unit mass as a function of the progenitor mass $M$. Here, we refer to the modified Salpeter-A IMF [12]

$$
\phi(M) \propto M^{-\zeta},
$$




\begin{tabular}{lccc}
\hline SN progenitor & $C\left[\times 10^{50} \mathrm{MeV}^{-1}\right]$ & $E_{0}[\mathrm{MeV}]$ & $\beta$ \\
\hline \hline $8.8 M_{\odot}$ & 2.77 & 90.80 & 2.58 \\
$11.2 M_{\odot}$ & 4.98 & 93.39 & 2.79 \\
$18 M_{\odot}$ & 16.0 & 120.2 & 2.42 \\
$25 M_{\odot}$ & 19.0 & 147.6 & 2.25 \\
$40 M_{\odot}$ & 1.43 & 174.4 & 1.81 \\
$70 M_{\odot}$ & 0.18 & 109.8 & 1.36 \\
\hline
\end{tabular}

Table 1: Fitting parameters for the SN ALP spectrum from the Primakoff process for different SN progenitors estimated for $g_{a \gamma}=10^{-11} \mathrm{GeV}^{-1}$ and $m_{a} \ll 10^{-11} \mathrm{eV}$.

with $\zeta=2.35$ for birth masses $M \gtrsim 0.5 M_{\odot}$, and $\zeta=1.5$ for $0.1 M_{\odot} \leq M \leq 0.5 M_{\odot}$. Consequently, the IMF-weighted ALP spectrum $\mathrm{d} N_{a}^{C C} / \mathrm{d} E_{a}$ of all CCSNe events can then be calculated as [13]

$$
\frac{\mathrm{d} N_{a}^{C C}}{\mathrm{~d} E_{a}}=\frac{\int \mathrm{d} M \phi(M) \frac{\mathrm{d} N_{a}}{\mathrm{~d} E}(M)}{\int_{8 M_{\odot}}^{125 M_{\odot}} \mathrm{d} M \phi(M)} .
$$

As shown in [13], a wide range of progenitor masses is expected to result in failed SN events, which depends on the assumed models and simulations. We hence incorporate this effect via the failed $\mathrm{SN}$ to CCSN ratio

$$
f_{\text {fail-SN }}=\frac{\int_{\Lambda_{\text {fail }}} \mathrm{d} M \phi(M)}{\int_{8 M_{\odot}}^{125 M_{\odot}} \mathrm{d} M \phi(M)}
$$

in our computation following the approach described in [10]. To this end, we consider different values for $f_{\text {fail-SN }}$ in line with the spread of theoretical expectations. The resulting uncertainty on the final DSNALPB spectrum is shown in the right panel of Fig. 1.

Once having reached the Milky Way's domain of influence the DSNALPB may be re-converted into gamma rays due to the transverse component of the Galactic magnetic field, $B_{T}$. The conversion probability, $P_{a \gamma}$, of an ALP travelling a distance $d$ may be expressed as [4]

$$
P_{a \gamma}=\left(\Delta_{a \gamma} d\right)^{2} \frac{\sin ^{2}\left(\Delta_{\mathrm{osc}} d / 2\right)}{\left(\Delta_{\mathrm{osc}} d / 2\right)^{2}}
$$

where $\Delta_{a \gamma} \equiv g_{a \gamma} B_{T} / 2$ and $\Delta_{\text {osc }}$ is a complex function of the local electron density of a medium $n_{e}$, $B_{T}$, the ALP energy and its mass [4]. However, for ALP masses $m_{a} \ll 10^{-11} \mathrm{GeV}^{-1}$ and $E \geq 10$ $\mathrm{MeV} P_{a \gamma}$ becomes practically energy-independent with $\Delta_{\mathrm{osc}} \rightarrow 0$. Eventually, the gamma-ray flux at the top of the Earth's atmosphere is given by

$$
\frac{\mathrm{d} \Phi_{\gamma}}{\mathrm{d} E}=\frac{1}{4 \pi d^{2}} \frac{\mathrm{d} N_{a}^{C C}}{\mathrm{~d} E_{a}} \times P_{a \gamma} .
$$

In what follows, we assume the Jansson and Farrar model of the Galactic magnetic field [14] with parameters updated to fit the measurements of the Planck satellite [15]. The spatial morphology of the gamma-ray flux of the DSNALPB hence follows the morphology of the Jansson and Farrar model as shown in Fig. 2. 


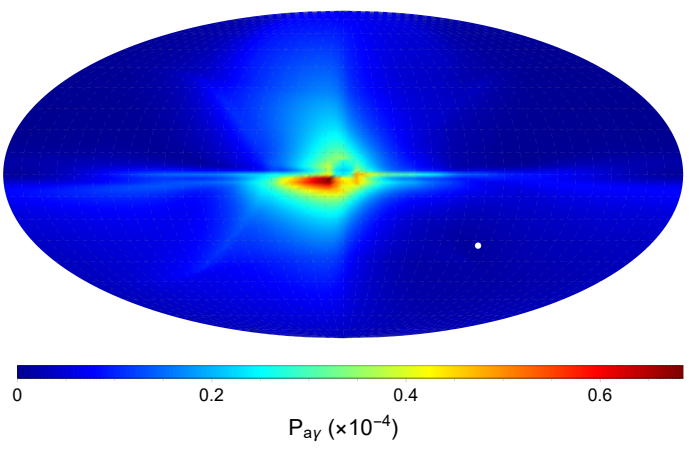

Figure 2: All-sky map (Galactic coordinate system) of the ALP-photon conversion probability $P_{a \gamma}$ with respect to the Jansson and Farrar Galactic magnetic field model [14] taking a point right at the boundary of the Milky Way as a reference point. This map represents an ALP at $E=50 \mathrm{MeV}$ with the coupling $g_{a \gamma}=3 \times 10^{13} \mathrm{GeV}^{1}$ and $m_{a} \ll 10^{11} \mathrm{eV}$. The white circle marks the position of SN 1987A.

\begin{tabular}{|l||c|c|}
\hline Data Set & $E \geq 200 \mathrm{MeV}$ & $E<200 \mathrm{MeV}$ \\
\hline \hline Reconstruction algorithm & \multicolumn{2}{|c|}{ Pass 8 } \\
\hline Event class & \multicolumn{2}{|c|}{ ULTRACLEANVETO } \\
\hline Event type & FRONT+BACK & PSF3 \\
\hline Energy range & $200 \mathrm{MeV}-500 \mathrm{GeV}$ & $50 \mathrm{MeV}-200 \mathrm{MeV}$ \\
\hline Time interval & 12 years (4th August $2008-3 \mathrm{rd}$ September 2020) \\
\hline ROI & \multicolumn{2}{c|}{ all sky } \\
\hline Zenith angle (applied to gtltcube) & \multicolumn{2}{c|}{$<90^{\circ}$} \\
\hline Time cuts filter & DATA_QUAL==1 \&\& LAT_CONFIG==1 \\
\hline HEALPix resolution & \multicolumn{2}{|c|}{$N_{\text {side }}=64$} \\
\hline energy binning & \multicolumn{2}{|c|}{ 30 logarithmically spaced bins } \\
\hline
\end{tabular}

Table 2: Data selection and preparation specifications

\section{Fermi-LAT analysis framework}

We employ a binned, template-based analysis based on a Poisson likelihood function utilising 12 years of Fermi-LAT data to constrain the extended large-scale signal of the DSNALPB. The LAT data selection criteria are listed in Tab. 2. All data preparation and simulation is conducted with the Fermi Science Tools ${ }^{1}$ (version 2.0.8). Since the DSNALPB spectrum peaks at the low-energy end of the LAT's sensitivity range, where its angular resolution becomes larger than a few degrees ${ }^{2}$, we divide the full data set into two parts - the cut being made at $E=200 \mathrm{MeV}$ - in order to optimise the analysis routine for both data sets independently. Eventually, both data sets are merged again in a joint-likelihood scan.

Regarding the construction of the likelihood function, we follow the approach applied by the Fermi-LAT collaboration in the context of their latest gamma-ray source catalogue iteration, the 4FGL [16]. They proposed to use a weighted log-likelihood function

$$
\ln \mathcal{L}_{w}(\boldsymbol{\mu} \mid \boldsymbol{n})=\sum_{i, p} w_{i p}\left(n_{i p} \ln \mu_{i p}-\mu_{i p}\right)
$$

${ }^{1}$ https://github.com/fermi-lat/Fermitools-conda

${ }^{2}$ c.f. https://www.slac.stanford.edu/exp/glast/groups/canda/lat_Performance.htm 
to incorporate the presence of instrumental systematic errors. Here, the likelihood function is subdivided into energy bins $i$ and spatial pixels $p$ of the input model data $\boldsymbol{\mu}$ and LAT data $\boldsymbol{n}$. Each pixel is weighted by a factor $w_{i p}$, which is derived via routines implemented in the Fermi Science Tools and based on the photon counts per pixel in the original LAT data set. Throughout the analysis, we assume a systematic uncertainty amplitude of $3 \%$ (the value utilised by the Fermi-LAT collaboration to derive the 4FGL source catalogue [16]).

Following the general rationale of template-based analyses, we gather a collection of astrophysical gamma-ray emission components $\boldsymbol{X}$ that are guaranteed to contribute to the gamma-ray sky. Thus, the model data are a linear combination of these templates

$$
\boldsymbol{\mu}=A^{\mathrm{ALP}} \boldsymbol{X}^{\mathrm{ALP}}+\sum_{X} \sum_{i} A_{i}^{X} X_{i}
$$

with the addition of the DSNALPB signal template $\boldsymbol{X}^{\text {ALP }}$ to which we assign a single, global normalisation parameter $A^{\mathrm{ALP}}$ that we aim to constrain. This construction relies on background normalisation parameters $A_{i}^{X}$ per energy bin so that these parameters can be varied independently of each other during a fitting step lending more freedom to each component in order to mitigate possible spectral uncertainties.

Our collection of astrophysical templates is the following:

- Interstellar emission (IE) from gas, dust and radiation fields along the Milky Way's disc. We select five distinct models to bracket the uncertainty on this component: Two models are taken from the the "1st Fermi LAT Supernova Remnant Catalog" [17]. The gamma-ray emission follows the distribution of pulsars in the Milky Way as reported in [18]. The typical height of the cosmic-ray propagation halo is set to $z=10 \mathrm{kpc}$, while the spin temperature of the interstellar medium is taken to be $T_{s}=150 / 1 \cdot 10^{5} \mathrm{~K}$. We label the model with $T_{s}=150 \mathrm{~K}$ "Lorimer I" and use it as the benchmark IE model. The other three models are the foreground models created by the Fermi-LAT collaboration to explore the spectrum of the isotropic gamma-ray background [19].

- Detected point-like and extended gamma-ray sources (PS) from the Fermi-LAT 4FGL source catalogue [16].

- Fermi Bubbles (FBs) according to the spectrum and spatial morphology derived in [20].

- LoopI represented by a geometrical model adopted from [21].

- Isotropic diffuse gamma-ray background (IGRB) using the implementation within the Fermi Science Tools.

- Emission from the Sun and the Moon likewise derived with the Fermi Science Tools based on the prepared LAT data sets.

General analysis rationale. We adopt and adapt the fitting strategy of the Fermi-LAT collaboration developed in the context of the optimisation of their diffuse gamma-ray background model ${ }^{3}$. This

\footnotetext{
${ }^{3}$ see https://fermi.gsfc.nasa.gov/ssc/data/analysis/software/aux/4fgl/Galactic_Diffuse_ Emission_Model_for_the_4FGL_Catalog_Analysis.pdf
} 
approach comprises an iterative all-sky fit in disjunct sky regions to determine the best-fit normalisations for each component from locations where they matter the most. Following this strategy, we first perform such an iterative fit to derive a baseline model with only the astrophysical components being present, i.e. $A^{\mathrm{ALP}}=0$. This baseline model is consequently utilised to determine the region of interest that yields robust upper limits on the ALP template normalisation. Upper limits are set based on the log-likelihood ratio test statistic TS $=2 \times\left(\ln \mathcal{L}_{w, 1}-\ln \mathcal{L}_{w, 0}\right)$, where $\ln \mathcal{L}_{w, 0}$ denotes the likelihood value minimised including the DSNALPB template while $\ln \mathcal{L}_{w, 1}$ is the value of the likelihood function for a particular value of $A^{\mathrm{ALP}}$ while profiling over the remaining astrophysical normalisation parameters. The $95 \%$ confidence level upper limits correspond to a TS-value of 2.71 . The statistical robustness is quantified via the compatibility of the TS-distribution obtained from the LAT data and the baseline model as data input $\boldsymbol{n}$.

\section{Results and conclusions}

The analysis procedure outlined in Sec. 3 has shown that irrespective of the LAT data set, the southern hemisphere of the gamma-ray sky at Galactic latitudes $b<-30^{\circ}$ yields statistically robust upper limits on the DSNALPB template normalisation. In Fig. 3, we show the obtained upper limits on the ALP-photon coupling constant $g_{a \gamma}$ with respect to the benchmark choices of the analysis, i.e. the Jansson and Farrar Galactic magnetic field model as well as the IE model "Lorimer I".

In this setting, we find an improvement (red band) of the upper limit on $g_{a \gamma}$ regarding the previous analysis [9] (dashed, light red line) that was solely based on the spectral shape of the ALPinduced gamma-ray flux. In numbers, we obtain - in the best possible scenario $-g_{a \gamma} \lesssim 2 \cdot 10^{-11}$ $\mathrm{GeV}^{-1}$ for ALP masses $m_{a} \ll 10^{-11} \mathrm{eV}$. However, the uncertainty of the constraints on $g_{a \gamma}$ is limited and the inclusion of the spatial morphology of the signal improves the limits even in the worst case compared to the spectral analysis standalone. It is thus essential for this kind of analysis to refine the currently existing models of the Milky Way's magnetic field structure since the constraining power is strongly correlated with the model quality.

Acknowledgements. C.E. is supported by the "Agence Nationale de la Recherche", grant $\mathrm{n}$. ANR-19-CE31-0005-01 (PI: F. Calore).

\section{References}

[1] M. Dine, in Theoretical Advanced Study Institute in Elementary Particle Physics (TASI 2000): Flavor Physics for the Millennium, 6, 2000. hep-ph/0011376.

[2] J. Jaeckel and A. Ringwald, Ann. Rev. Nucl. Part. Sci. 60 (2010) 405-437, [1002.0329].

[3] P. Arias, D. Cadamuro, M. Goodsell, J. Jaeckel, J. Redondo and A. Ringwald, JCAP 06 (2012) 013, [1201. 5902].

[4] G. Raffelt and L. Stodolsky, Phys. Rev. D 37 (Mar, 1988) 1237-1249.

[5] G. G. Raffelt, Phys. Rev. D 33 (1986) 897.

[6] G. G. Raffelt, Lect. Notes Phys. 741 (2008) 51-71, [hep-ph/0611350].

[7] T. Fischer, S. Chakraborty, M. Giannotti, A. Mirizzi, A. Payez and A. Ringwald, Phys. Rev. D 94 (2016) 085012, [1605.08780]. 


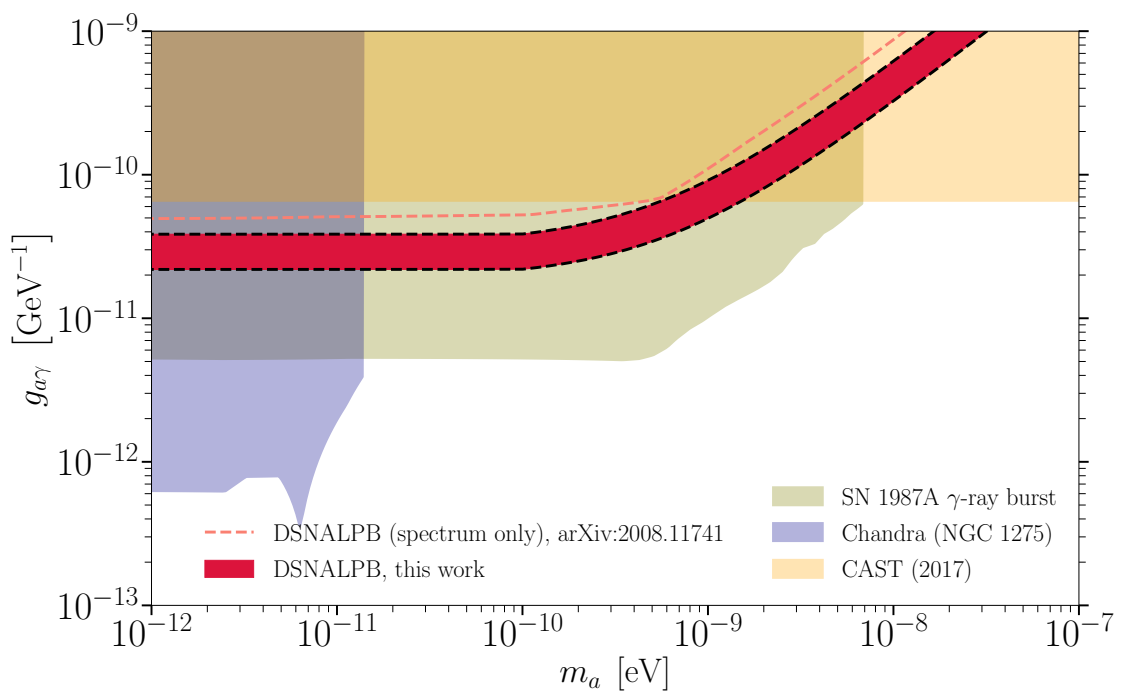

Figure 3: $95 \%$ confidence level upper limits on the ALP-photon coupling constant $g_{a \gamma}$ using 12 years of Fermi-LAT data prepared according to the description in Sec. 3 and assuming the Jansson and Farrar Galactic magnetic field model as well as "Lorimer I" as IE model. The red band displays the expected uncertainty on the upper limits due to the different ratios of failed and successful CCSNe as summarised in the right panel of Fig. 1. The dashed, light red line denotes the constraints derived in a previous work that was solely based on the spectral shape of the DSNALPB [9]. As a means to compare the constraining power of this ALP-induced signal, we display a selection of current bounds on $g_{a \gamma}$ from other experiments and events.

[8] A. Payez, C. Evoli, T. Fischer, M. Giannotti, A. Mirizzi and A. Ringwald, JCAP 02 (2015) 006, [1410 . 3747 ].

[9] F. Calore, P. Carenza, M. Giannotti, J. Jaeckel and A. Mirizzi, Phys. Rev. D 102 (2020) 123005, [2008. 11741].

[10] A. Priya and C. Lunardini, JCAP 11 (2017) 031, [1705.02122].

[11] Planck collaboration, N. Aghanim et al., Astron. Astrophys. 641 (2020) A6, [1807.06209].

[12] I. K. Baldry and K. Glazebrook, Astrophys. J. 593 (2003) 258-271, [astro-ph/0304423].

[13] D. Kresse, T. Ertl and H.-T. Janka, Astrophys. J. 909 (2021) 169, [2010.04728].

[14] R. Jansson and G. R. Farrar, ApJ 757 (Sept., 2012) 14, [1204.3662].

[15] Planck collaboration, R. Adam et al., Astron. Astrophys. 596 (2016) A103, [1601.00546].

[16] Fermi-LAT collaboration, S. Abdollahi et al., Astrophys. J. Suppl. 247 (2020) 33, [1902 . 10045$].$

[17] Fermi-LAT collaboration, F. Acero et al., Astrophys. J. Suppl. 224 (2016) 8, [1511.06778].

[18] D. R. Lorimer et al., Mon. Not. Roy. Astron. Soc. 372 (2006) 777-800, [astro-ph/0607640].

[19] Fermi-LAT collaboration, M. Ackermann et al., Astrophys. J. 799 (2015) 86, [1410 . 3696].

[20] Fermi-LAT collaboration, M. Ackermann et al., Astrophys. J. 840 (2017) 43, [1704.03910].

[21] M. Wolleben, Astrophys. J. 664 (2007) 349-356, [0704.0276]. 